\title{
Isolation of a Human Epidermal CDNA Corresponding to the 180-kD Autoantigen Recognized by Bullous Pemphigoid and Herpes Gestationis Sera
}

\author{
Immunolocalization of This Protein to the Hemidesmosome
}

\author{
Luis A. Diaz, Harry Ratrie III,* William S. Saunders, * Shozo Futamura, H. Luis Squiquera, \\ Grant J. Anhalt, * and George J. Giudice \\ Departments of Dermatology, Medical College of Wisconsin, Milwaukee, Wisconsin 53226; *Johns Hopkins University, \\ Baltimore, Maryland 21205; and the Veterans Administration Medical Center, Milwaukee, Wisconsin 53193
}

\begin{abstract}
Autoantibodies present in the sera of patients with bullous pemphigoid (BP) bind to the basement membrane zone of normal human skin and commonly recognize two epidermal proteins, the BP240 and BP180 antigens. Two BP antigen cDNA clones from a lambda gt11 human keratinocyte library have been identified on the basis of reactivity with a BP serum. The fusion protein (FP) produced by one clone immunoadsorbed autoantibodies, which specifically recognized the BP180 antigen, showing no cross-reactivity with BP240 by immunoblot analysis. The FP produced by the second clone immunoadsorbed autoantibodies which specifically reacted with the BP240 epidermal antigen. Northern blot analysis demonstrated that the BP180 and BP240 antigens are encoded by distinct RNA transcripts with lengths of 6.0 and $8.5 \mathrm{~kb}$, respectively. Immunoblot analysis of the BP180 lysogen extract identified a 135-kD FP which was recognized by 7 of 16 BP sera and 7 of 8 herpes gestationis sera. A rabbit antiserum prepared against the lysogenic BP180 FP specifically recognized the BP180 antigen from human epidermal extracts by immunoblotting, labeled the BMZ by indirect immunofluorescence, and bound to human epidermal hemidesmosomes by immuno-electron microscopy. These results indicate that the BP180 antigen recognized by BP and herpes gestationis autoantibodies is a unique hemidesmosomal polypeptide, distinguishable from the BP240 antigen. (J. Clin. Invest. 1990. 86:1088-1094.) Key words: autoimmunity • autoantibody • keratinocyte $\cdot$ basement membrane
\end{abstract}

\section{Introduction}

Bullous pemphigoid (BP) ${ }^{1}$ is an autoimmune skin disease of unknown etiology characterized by subepidermal vesicles and autoantibodies against components of the cutaneous basement

Presented in abstract form on 29 April 1989 at the annual meeting of The Society for Investigative Dermatology, Washington, DC.

Address correspondence and reprint requests to Dr. George J. Giudice, Department of Dermatology, Medical College of Wisconsin, 8701 Watertown Plank Road, Milwaukee, WI 53226.

Received for publication 16 February 1990 and in revised form 24 April 1990.

1. Abbreviations used in this paper: BMZ, basement membrane zone; BP, bullous pemphigoid; EM, electron microscopy; EPF, endemic pemphigus foliaceus; FP, fusion protein; HG, herpes gestationis; IF, immunofluorescence; IPTG, isopropyl-beta-D-thiogalactopryanoside; NC, nitrocellulose; NHS, normal human serum; PV, pemphigus vulgaris.

J. Clin. Invest.

(c) The American Society for Clinical Investigation, Inc.

0021-9738/90/10/1088/07 $\$ 2.00$

Volume 86, October 1990, 1088-1094 membrane zone (BMZ) (1). BP skin lesions show a clean separation of the epidermis from the dermis through the lamina lucida of the BMZ (2). In 1967, Jordon et al. (3), using an immunofluorescence (IF) assay, detected BP autoantibodies bound to the BMZ of lesional skin, as well as circulating in the sera of these patients. Early ultrastructural studies of the tissue-bound autoantibodies showed the immunoreactants to be localized in the lamina lucida of the BMZ (4). Recent reports have demonstrated that circulating $\mathrm{BP}$ autoantibodies bind specifically to the hemidesmosome (5-8), an organelle which is thought to be involved in the adherence of the epidermal basal cell to the dermis. The relevance of either tissue-bound or circulating autoantibodies with respect to the mechanism of blister formation in BP patients remains unclear.

Several studies have been reported on the characterization of BP antigens. Stanley and collaborators have shown that BP sera bind specifically to a single epidermal polypeptide of 230/240 kD (referred to here as BP240 antigen) by both immunoblotting and immunoprecipitation procedures $(9,10)$. Our group and others have found that, in addition to the BP240 antigen, BP sera also recognize lower molecular weight epidermal antigens $(11,12)$. One such antigen is the $180-\mathrm{kD}$ protein (referred to here as BP180 antigen), which is recognized by $\sim 50 \%$ of all $\mathrm{BP}$ sera and the majority of Herpes Gestationis (HG) sera (13). HG is an idiopathic subepidermal disease of pregnancy which exhibits several features common to BP, including the presence of anti-BMZ autoantibodies (14).

Using a BP serum containing autoantibodies against the BP240 antigen, Stanley et al. (15) have recently identified a 2-kb human keratinocyte cDNA that encodes epitope(s) recognized by these autoantibodies. The BP cDNA encoded a fusion protein (FP) which immunoadsorbed BP autoantibodies directed against the BP240 antigen from the respective BP serum. Northern blot analysis has shown that this cDNA hybridizes with a 9-kb keratinocyte transcript.

In this report we describe the characterization of a 1-kb cDNA purified from a keratinocyte cDNA library which encodes an FP recognized by BP and HG autoantibodies against the BP180 antigen. A rabbit antiserum prepared against the BP180 cDNA-encoded FP reacted with the BP180 epidermal antigen by immunoblotting and labeled the epidermal hemidesmosome by immuno-electron microscopy (immuno-EM). Northern blot analysis shows hybridization of the BP180 cDNA with a 6.0-kb RNA transcript which is distinct from the 8.5-kb mRNA of the BP240 antigen. This study indicates that the BP240 and BP180 antigens are distinct hemidesmosomal proteins.

\section{Methods \\ Sources of sera. Sera from BP patients $(n=16), \mathrm{HG}(n=8)$, pemphigus vulgaris (PV) $(n=5)$, endemic pemphigus foliaceus (EPF) $(n=10)$,}


and normal human donors (NHS) $(n=10)$ were used in this investigation. The HG sera used in this study were the same as those used in a previous investigation (13). All BP sera possessed anti-BMZ autoantibodies displaying titers above $1: 80$ by indirect IF procedures. Using immunoblotting techniques the BP240 antigen was labeled by the majority of the BP sera tested, while the BP180 antigen was recognized by half of the BP sera and by all HG sera (results which are consistent with those previously reported) (13). PV and EPF sera exhibited, by indirect IF, the characteristic epidermal intercellular staining produced by the respective autoantibodies, while the normal human serum (NHS) showed no reactivity with epidermal antigens.

Immunofluorescence and immunoblotting. Reactivity of human sera (or rabbit anti-FP) against the BMZ and ICS of human skin cryosections was investigated by routine indirect IF procedures $(5,13)$. Cytospin-fixed trypsin-dissociated human epidermal basal cells were also used to test BP autoantibodies by indirect IF following procedures reported elsewhere (5). FITC-antihuman IgG and FITC-antirabbit IgG were from Cappel Laboratories (West Chester, PA).

Extraction and electrophoretic separation of epidermal proteins has been described elsewhere $(11,13)$. Briefly, heat-separated human epidermal sheets were extracted by homogenization using a 1\% SDS, $0.01 \mathrm{M}$ Tris buffer, $\mathrm{pH} 7.6$ in the presence of $10 \mathrm{mM}$ EDTA, $1 \mathrm{mM}$ PMSF, and $10 \mu \mathrm{g} / \mathrm{ml}$ of each of the following protease inhibitors: leupeptin, antipain, chymostatin, and pepstatin. Reduced SDS-PAGE electrophoresis was carried out using either 7 or $7-15 \%$ gradient gels. Similar extraction and electrophoretic procedures were used to solubilize and separate lysogenic extracts containing the putative BP180 FP.

Immunoblotting procedures performed in this study have been described previously by Labib et al. (11) and Morrison et al. (13). SDS-PAGE fractionated proteins were transferred to nitrocellulose (NC) paper and then probed with human or rabbit antisera at a dilution of $1: 200$ in $4 \%$ PTX buffer $(0.01 \mathrm{M}$ phosphate, $\mathrm{pH} 7.5,0.2 \%$ Triton X-100, $0.15 \mathrm{M} \mathrm{NaCl}$, and $1 \mathrm{mM}$ EGTA). Bound antibodies were demonstrated using ${ }^{125} \mathrm{I}$-Staphylococcus aureus protein A, instead of the enzymatic method used by Labib and Morrison. Immunoreactive protein bands recognized by antibodies were visualized by autoradiography using Kodak XAR5 film (Kodak Laboratory and Specialty Chemicals, Rochester, NY) with DuPont Lightning Plus intensifying screens (DuPont Co., Wilmington, DE).

Immunoelectron microscopy. Immuno-EM procedures were performed as previously reported (5-6) using peroxidase-conjugated goat antihuman IgG (Cappel Laboratories) and 3,3'-diaminobenzidine (Sigma Chemical Co., St. Louis, MO) as the indicator. Ultrathin sections were observed by electron microscopy without counterstaining.

Screening of a keratinocyte cDNA library with BP serum. A high titer BP serum (indirect IF titer 1:1280) containing autoantibodies against both the BP180 and BP240 epidermal antigens was used at the dilution of 1:100 to screen a human keratinocyte lambda-gt $11 \mathrm{cDNA}$ library (Clontech Laboratories, Palo Alto, CA) according to the method of Young and Davis (16). LacZ-directed expression was induced by first incubating the cultures at $42^{\circ} \mathrm{C}$ for $3-4 \mathrm{~h}$ followed by overlaying an NC filter (previously soaked in $10 \mathrm{mM}$ isopropyl-betaD-thiogalactopyranoside [IPTG]) onto the plaques at $37^{\circ} \mathrm{C}$ for an additional 3-4 h. During this incubation, beta-galactosidase FP is produced by the lytically infected cells and immobilized on the NC paper. After the filters were blocked with 4\% BSA in PTX buffer, they were incubated overnight with an appropriate dilution of BP serum. The NC filters were washed thoroughly in GB buffer $(50 \mathrm{mM}$ triethanolamine, pH 7.4, $0.5 \%$ Triton X-100, $0.1 \mathrm{M} \mathrm{NaCl}, 2 \mathrm{mM}$ EDTA, and $0.1 \%$ SDS $)$ and incubated with ${ }^{125} \mathrm{I}$-labeled protein $\mathrm{A}\left(2 \times 10^{5} \mathrm{cpm} / \mathrm{ml}\right)$. Unbound radiolabeled protein A was removed by washing in GB as above. The immunoreactive clones were detected by autoradiography. To assure plaque purity, repeated rounds of screening of each clone were performed.

Affinity purification of BP autoantibodies against the recombinant phage plaques. An agar plate containing Y 1090 Escherichia coli lawns infected with plaque-pure recombinant phages was overlaid with an NC filter soaked in $10 \mathrm{mM}$ IPTG and incubated at $37^{\circ} \mathrm{C}$ for $2 \mathrm{~h}$. The filters were then removed, blocked with 4\% BSA in PTX for $1 \mathrm{~h}$, and incubated with the same BP serum used to screen the library. Nonspecifically bound antibodies were removed by five washes with PTX. Specifically bound BP autoantibodies were eluted from the NC filters using $0.2 \mathrm{M}$ glycine $\mathrm{HCl}, \mathrm{pH} 2.8$, and immediately neutralized with 1 M Tris, pH 9.5. The eluted antibodies were dialyzed with Tris-buffered saline (TBS), concentrated by ultrafiltration, and characterized by IF and immunoblotting procedures.

Production of CDNA-encoded BP fusion protein by lysogens. Lambda-gt 11 lysogens were generated in $E$. coli Y 1089 using plaquepurified lambda-BP180 phages following procedures described by Earnshaw et al. (17). Liquid cultures of BP180 lysogens were induced to enter into the lytic phase by raising the temperature to $45^{\circ} \mathrm{C}$ for 15 min followed by a 2 -h incubation at $37^{\circ} \mathrm{C}$ in the presence of $10 \mathrm{mM}$ IPTG as described by Miller (18). A time course study was performed in order to determine the optimal incubation time for the production of the BP FP. BP180 lysogens were harvested by centrifugation and the FP extracted and analyzed by SDS-PAGE and immunoblotting as described above.

Immunization of rabbits with BP180 fusion protein. Total extracts of the BP180 lysogen in 1\% SDS were fractionated by SDS-PAGE and electrotransferred to NC paper as described above. A horizontal strip of NC containing the 135-kD BP180 FP was cut out, frozen in liquid nitrogen, and pulverized with a mortar and pestle. The powder was resuspended in TBS, mixed with complete Freund adjuvant, and injected subcutaneously into a rabbit as reported elsewhere (19). Booster doses were given every 2 wk. The rabbit anti-BP180 FP serum was subsequently used for immunocytochemical and immunoblot procedures.

Subcloning of the lambda cDNA inserts. Recombinant bacteriophages, lambda-BP240, and lambda-BP180 were isolated from largescale liquid lysates of infected LE392 cells, and the phage DNA was extracted according to the procedure of Ausubel et al. (20). The cDNA inserts were obtained by digestion of the recombinant lambda DNA with Eco RI and were purified by gel electrophoresis using SeaPlaque low melting temperature agarose (FMC Corp., Rockland, ME). The BP240 and BP180 cDNA inserts were ligated into the Eco RI site of the Bluescript $\mathrm{KS}^{+}$vector (Stratagene Inc., La Jolla, $\mathrm{CA}$ ) and the resulting constructs will be referred to as pBKS-BP240 and pBKS-BP180, respectively.

Northern blot analysis. Northern analysis was performed as previously described (21). Total RNA extracted from a primary culture of human keratinocytes was fractionated by formaldehyde/agarose gel electrophoresis (35 $\mu \mathrm{g}$ total RNA/lane) and transferred to NC. The RNA blots were prehybridized at $42^{\circ} \mathrm{C}$ in a solution of $50 \%$ formamide, $20 \mathrm{mM}$ Hepes, pH 7.4, $5 \times$ SSC, $1 \times$ Denhardt's solution, 10 $\mu \mathrm{g} / \mathrm{ml}$ yeast tRNA, and $10 \mu \mathrm{g} / \mathrm{ml}$ sonicated, boiled salmon sperm DNA. ${ }^{32} \mathrm{P}$-labeled cRNA probes were prepared in the following manner. The recombinant plasmids, pBKS-BP240 and pBKS-BP180, were linearized and the appropriate RNA polymerase, T3 or T7, was used to generate a radiolabeled RNA strand complementary to the DNA template strand. The radiolabeled probes were added to the above hybridization solution at $4 \times 10^{5} \mathrm{cpm} / \mathrm{ml}$ and then hybridized with the fractionated RNA at $42^{\circ} \mathrm{C}$ overnight. The blots were washed in $0.1 \times$ SSC, $0.1 \%$ SDS at $70^{\circ} \mathrm{C}$, and exposed to Kodak XAR5 film using Lightning Plus intensifying screens (DuPont Co.). In certain cases, as indicated, the radiolabeled RNA blots were treated with $0.1 \mu \mathrm{g} / \mathrm{ml}$ RNase A in $2 \times$ SSC at room temperature for $15 \mathrm{~min}$, and subsequently washed in $0.1 \times \mathrm{SSC}, 0.1 \% \mathrm{SDS}$ at $50^{\circ} \mathrm{C}$.

\section{Results}

Identification of CDNA clones that encode epitopes recognized by BP autoantibodies. After screening $\sim 5 \times 10^{5}$ clones of the human keratinocyte cDNA expression library with the BP serum containing autoantibodies against the BP240 and BP180 antigens, several immunoreactive clones were detected, 
two of which have been further characterized. BP autoantibodies were affinity purified against the FP of each of these clones and were subsequently analyzed by immunoblot and indirect IF. As shown in Fig. 1, lane 1, the FP of one clone, lambdaBP240, immunoadsorbed antibodies directed against the BP240 antigen. BP autoantibodies purified against the products of the second cDNA clone, lambda-BP180, specifically recognized the BP180 antigen by immunoblot analysis (Fig. 1, lane 2). Both of these affinity-purified autoantibody preparations stained the BMZ of human skin by indirect IF and showed no cross-reactivity with other epidermal antigens by immunoblotting.

Restriction enzyme analysis of these clones revealed DNA inserts of $1.6 \mathrm{~kb}$ for the lambda-BP240 cDNA and $1.0 \mathrm{~kb}$ for the lambda-BP180 cDNA.

Northern blot analysis. Radiolabeled, strand-specific cRNA probes generated for both of the BP cDNAs were hybridized with human keratinocyte RNA; the resulting autoradiogram is shown in Fig. 2. The BP180 cRNA probe, which was prepared using T3 RNA polymerase, was found to hybridize with two bands at 6.0 and $4.9 \mathrm{~kb}$, the smaller band co-migrating with the 28S rRNA band (lane 1 ). Weak hybridization with a 4.9-kb band was also seen with the BP180 probe generated by the T7 RNA polymerase (lane 2 ). The hybridization with the 4.9-kb band by both of the BP180 probes was selectively eliminated by treatment of the blots with RNase A, suggesting that this observed hybridization was due to short stretches of homology with an abundant RNA species, such as 28S rRNA.

The BP240 cRNA probe generated by T7 RNA polymerase hybridized with a band of $\sim 8.5 \mathrm{~kb}$ (Fig. 2, lane 4 ). The opposite strand showed no hybridization demonstrating the low level of nonspecific hybridization (Fig. 2, lane 3).

Analysis of fusion protein produced by the BP-180 lysogen. Lysogenic Y $1089 E$. coli bearing the lambda-BP180 prophage produced FP after induction of the lytic phase in the presence of IPTG. As shown in Fig. 3, BP180 lysogen expressed a 135kD FP in a time-dependent fashion. Optimal amounts of this protein were produced after $2 \mathrm{~h}$ of IPTG incubation. The FP was not synthesized at $30^{\circ} \mathrm{C}$ or in the absence of IPTG. Im-

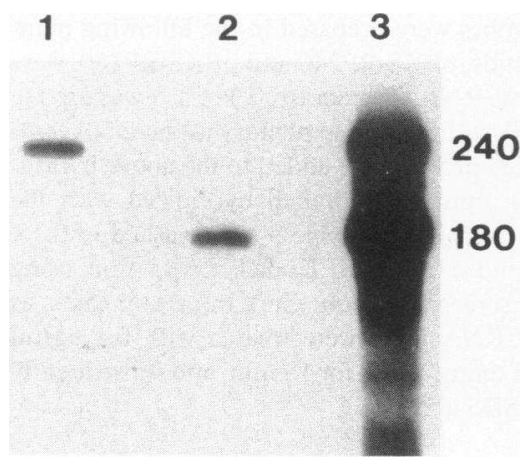

Figure 1. Fusion proteins produced by clones, lambda-BP 180 and lambda-BP240, immunoadsorb autoantibodies specific for the BP180 and BP240 antigens, respectively. The IPTG-inducible products of the two recombinant cDNA clones, lambda-BP240 and lambda-BP180, were immobilized on NC

and used as an immunoadsorbant to affinity purify autoantibodies from a BP serum. An immunoblot containing a PAGE-fractionated total protein extract from human epidermis was then labeled with the following antibody preparations: BP autoantibodies eluted from immobilized lambda BP240 plaques (lane 1); BP autoantibodies eluted from immobilized lambda BP180 plaques (lane 2); and unfractionated BP serum (lane 3).

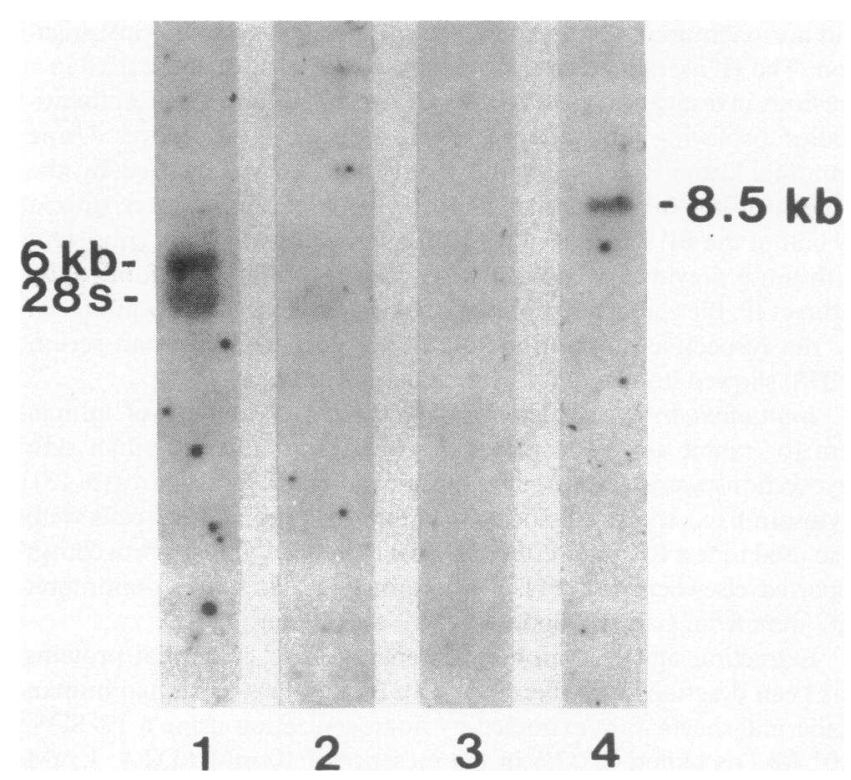

Figure 2. The BP180 and BP240 antigens are encoded by distinct RNA transcripts which are 6.0 and $8.5 \mathrm{~kb}$ in length, respectively. Northern blot analysis of total RNA from cultured human keratinocytes ( $35 \mu \mathrm{g} / \mathrm{lane}$ ) probed with: pBKS-BP180 cRNA probes (lanes 1 and 2) and pBKS-BP240 cRNA probes (lanes 3 and 4) generated by in vitro transcription using either the T3 RNA polymerase (lanes 1 and 3), or the T7 RNA polymerase (lanes 2 and 4 ). Lane 1 shows nonspecific hybridization of the BP180 probe with the $28 \mathrm{~S}$ rRNA band.

munoblot analysis demonstrated that the BP180 FP was recognized by the BP serum, which was used to screen the library (Fig. 3), and by a rabbit anti-beta galactosidase serum.

Immunoblot analyses were performed on the 135-kD BP180 FP using a panel of sera from BP, HG, PV, and EPF patients as well as NHS. Positive reactions were detected with 7 out of 16 BP sera and with 7 of $8 \mathrm{HG}$ sera. No reaction was obtained with $5 \mathrm{PV}, 10 \mathrm{EPF}$, and 10 normal control sera. A sample of these results are shown in Fig. 4 . Fig. $4 \mathrm{~A}$ shows a set of immunoblots containing a total protein extract from the BP180 lysogen labeled with the sera from seven BP patients. A positive reaction with the FP can be seen in all seven lanes. Fig. $4 \mathrm{~B}$, shows immunoblots of the BP180 lysogenic extract labeled with eight different HG sera (lanes $1-8$ ) and one NHS control (lane 9). A positive reaction with the FP is clearly detectable in lanes $1-7$. The very weak reaction obtained by the HG serum in lane 8 was recorded as a negative. The absence of a reaction demonstrated in lane 9 is representative of the results obtained with the PV, EPF, and normal human sera.

Analysis of a rabbit antiserum directed against the lambda-BP180 fusion protein. An antiserum generated by immunizing a rabbit with the BP180 FP was shown to react specifically with the BP180 antigen from a human epidermal extract by immunoblotting (Fig. $5 \mathrm{~A}$ ). No reactivity with the BP240 antigen was detected on these blots.

This rabbit antiserum also stained the BMZ of human skin in a linear pattern by indirect IF (Fig. 5 B). Trypsin-dissociated epidermal basal cells bound the rabbit antibodies producing "polar" caps by indirect IF. Ultrathin sections of human skin treated with the rabbit anti BP180 FP antibodies showed spe- 


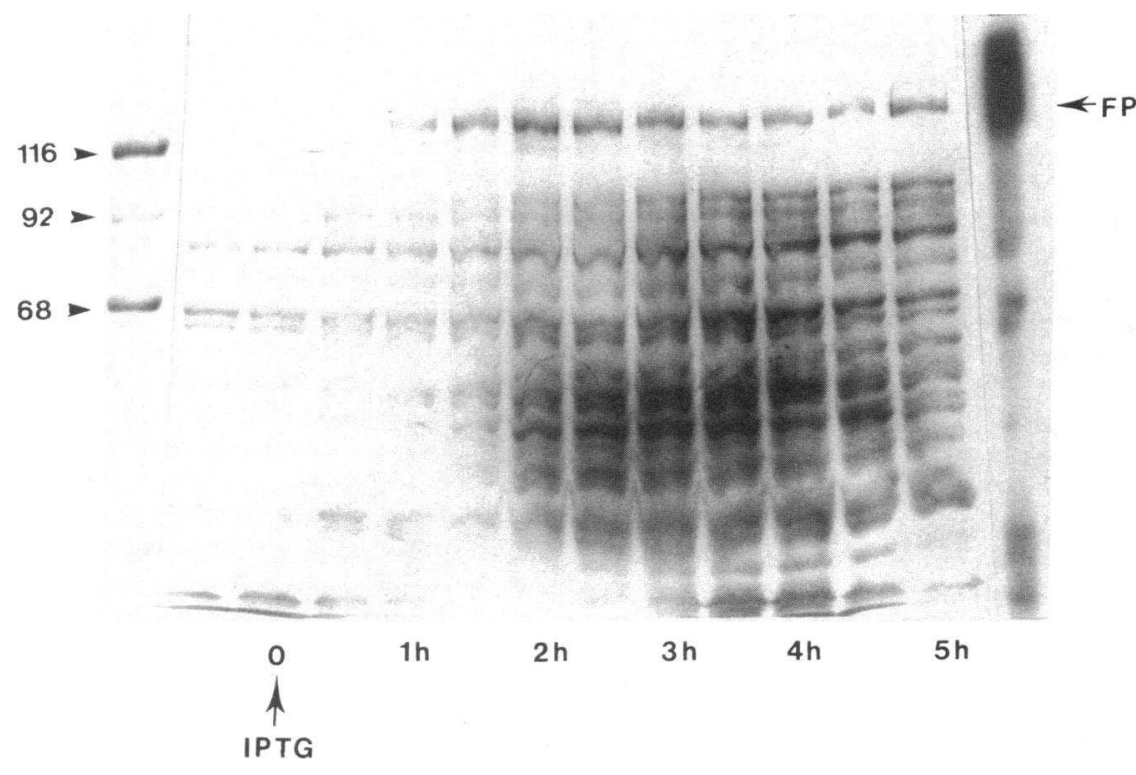

Figure 3. Time course of induction of fusion protein made by the BP 180 lysogen. Total SDS extracts of the BP180 lysogen were prepared at half-hour intervals following IPTG treatment (see designations at bottom of lanes) and analyzed by SDS-PAGE. Maximal levels of the BP180 fusion protein (designated " $F P$ ") are detected $\sim 2 \mathrm{~h}$ after IPTG addition. The lane at the far right is an immunoblot of the same lysogen extract taken at $2 \mathrm{~h}$ post-IPTG and probed with an unfractionated BP serum showing reactivity with the induced BP180 FP. The first lane contains molecular weight markers as indicated at left (in kilodaltons). The 135-kD BP180 FP also reacted with antibeta galactosidase antibodies as expected (data not shown).

cific labeling of the hemidesmosomes by immuno-EM analysis (Fig. $5 C$ ).

\section{Discussion}

A lambda-gt 11 human keratinocyte cDNA library was screened with a BP serum in order to identify cDNAs which encode epitopes recognized by BP autoantibodies. Clones were selected on the basis of antibody reactivity and were further characterized by immunologically analyzing the cDNA-encoded FP. The initial characterization involved an immunoblot analysis of BP autoantibodies affinity purified against the products of the recombinant bacteriophage clones. This analysis demonstrated that clone lambda-BP240 encodes a set of epitopes which are present on the BP240 epidermal antigen, as indicated by the single $240-\mathrm{kD}$ immunoreactive band shown in Fig. 1, lane 1. Furthermore, this analysis demonstrated that the BP240 epitopes recognized by these affinity-purified autoantibodies are not found on the BP180 antigen. In a similar analysis, clone lambda-BP180 was shown to express a set of epitopes which are present on the BP180 epidermal antigen, but not found on the BP240 antigen. This demonstration that the epitopes present on the BP180 antigen are not merely a subset of BP240 epitopes indicates that the BP180 antigen is not a breakdown product of BP240.

Further clarification of the biosynthetic relationship between the two major BP antigens was obtained by the Northern blot analysis which demonstrated that BP240 and BP180 antigens are encoded by distinct RNA transcripts. The BP240 cRNA probe hybridized with a single RNA transcript of $\sim 8.5$ $\mathrm{kb}$, while the BP180 probe hybridized with a $6.0-\mathrm{kb}$ transcript from human keratinocytes. No cross-hybridization between these two transcripts was detected. The sizes of these hybridizing transcripts are well within the range of expected sizes based on the apparent molecular weights of the BP180 and BP240 epidermal polypeptides. It is clear from the above data that the BP240 and BP180 antigens do not arise as a posttranslational processing of a single translation product. A comparison of the nucleotide sequence of a portion of the lambda-BP2 40 cDNA (Giudice, G., P. Elias, and L. Diaz, unpublished data) with the sequence of the BP antigen clone reported by Stanley et al. (15), confirms that these two cDNAs have been generated from the same RNA transcript. Our estimate of the length of the BP240 mRNA $(8.5 \mathrm{~kb})$ is in general agreement with the previously calculated size of this transcript (15).

The lambda-BP180 cDNA clone was further characterized by analyzing the cDNA-encoded FP using a variety of immunological techniques. In order to produce large amounts of this FP, a BP180 lysogen was generated by infecting Y 1089 E. coli with the phages from clone lambda-BP180. SDS-PAGE and immunoblot analysis of the BP180 lysogen extract identified a 135-kD IPTG-inducible FP which reacted strongly with the BP serum that was used to screen the cDNA library. As expected, the BP180 FP was also recognized on an immunoblot, by a rabbit antibody directed against beta-galactosidase. This confirms that the BP180 cDNA was inserted into the lambdagt 11 vector in such a way that the BP180 reading frame was in register with that of the lac $\mathrm{Z}$ coding region. The beta-galactosidase portion of such an FP generated by lambda gt 11 accounts for $114 \mathrm{kD}$ of its total size. Based on an apparent molecular weight of $135 \mathrm{kD}$ for the lambda BP180 FP, the cDNA-encoded portion should correspond to $\sim 21,000 \mathrm{D}$. A peptide of this size requires a transcript length of $\sim 600$ bases, suggesting that $\sim 60 \%$ of the $1-\mathrm{kb}$ BP1 $180 \mathrm{cDNA}$ exists in the form of an open reading frame.

The lysogenic BP180 FP was recognized on immunoblots by $44 \%$ of BP sera and $88 \%$ of HG sera. No reactivity was detected with a variety of control sera, which included PV, EPF, and NHS. The reactivity of BP and HG sera with the lysogenic BP180 FP closely paralleled their reactivity with the 180-kD epidermal BP antigen. These data confirmed a previously published report that BP and HG autoantibodies recognize a common epidermal polypeptide, the BP180 antigen (13). The present analysis further demonstrates that a set of epitopes, which are recognized by both BP and HG autoantibodies, are clustered within the small region of the BP180 antigen which is encoded by the lambda-BP 180 cDNA, a stretch of $\sim 200$ amino acids. It is interesting to note that the BP180 epidermal antigen appears to be the primary target of HG autoantibodies, whereas in BP, the autoantibody response 


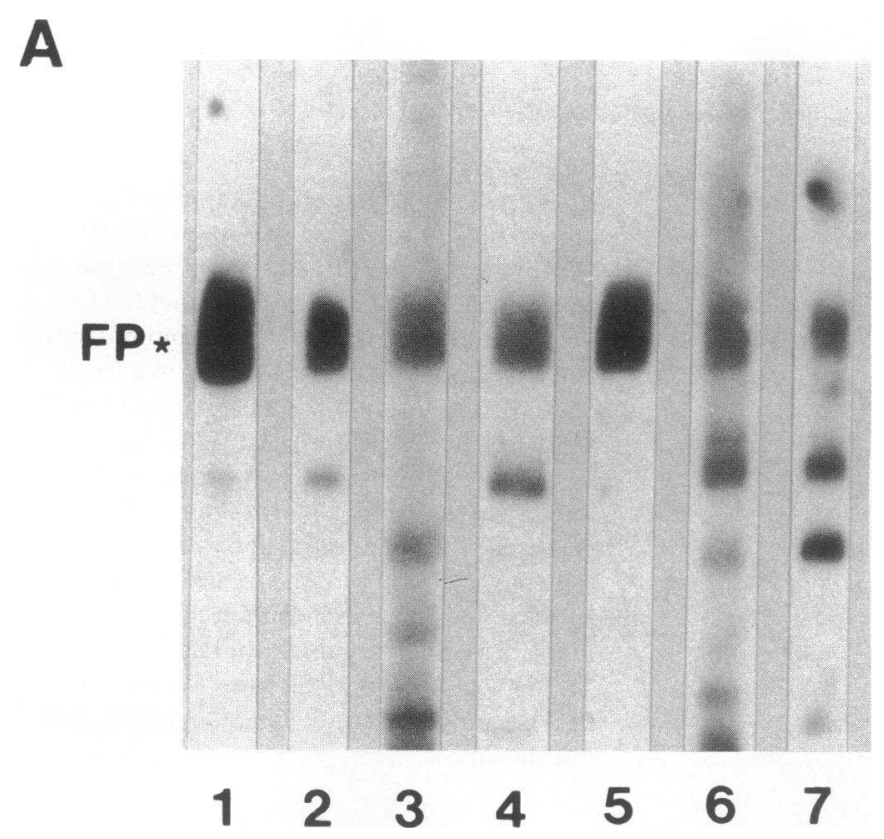

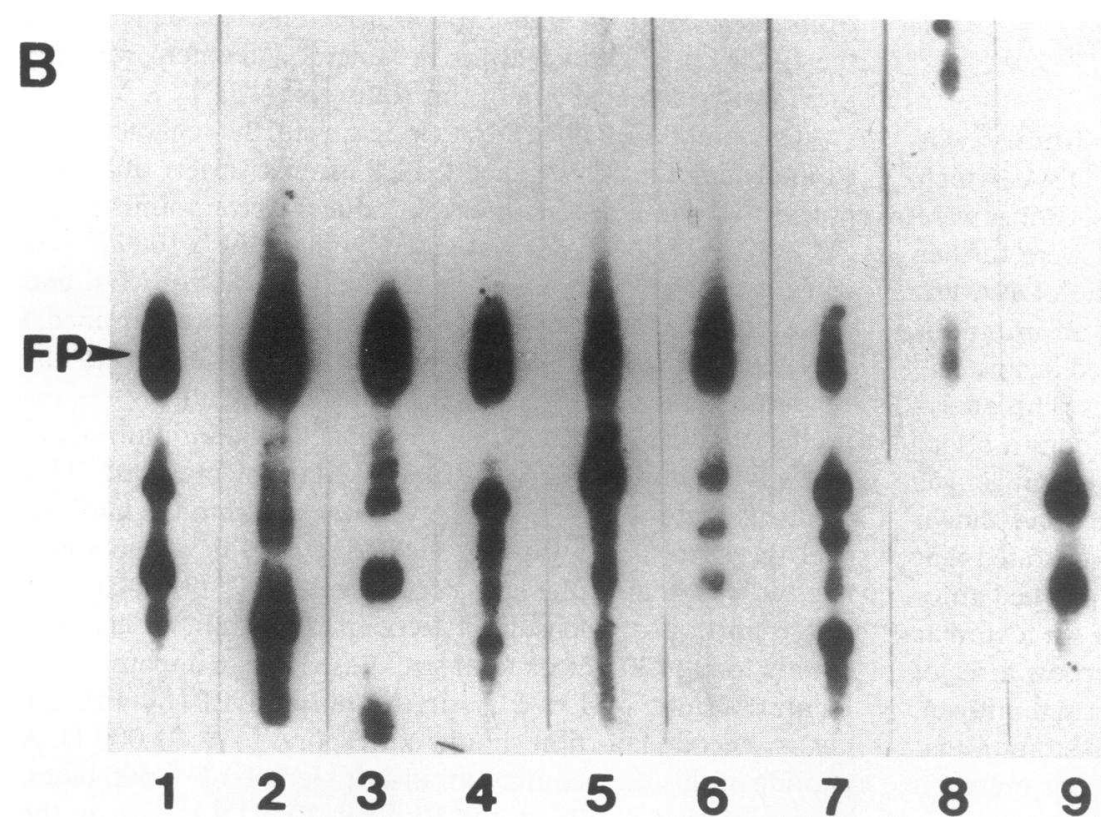

Figure 4. Immunoblot analysis of the lambdaBP180 fusion protein using BP and HG sera. Immunoblots containing a total protein extract from the IPTG-treated BP180 lysogen were labeled with the unfractionated serum from seven different BP patients $(A$, lanes $1-7)$, with eight different HG sera $(B$, lanes $1-8)$, and with a normal human serum $(B$, lane 9$)$. A positive reaction with the lambda-BP180 FP can be seen in all lanes of $A$ and in lanes $1-7$ of $B$. is mainly directed to the BP240 antigen. The relevance of these findings in the immunopathology of these diseases remains unknown.

Further evidence that the lambda-BP180 clone encodes a portion of the BP180 epidermal antigen was obtained by preparing and analyzing a rabbit antiserum directed against the lysogenic BP180 FP. Immunoblot analysis demonstrated that this anti-FP antiserum specifically recognized the BP180 antigen from a total SDS extract of normal human epidermis. The set of BP180 epitopes recognized by this rabbit serum are not present on the BP2 40 antigen, as evidenced by the absence of immunoblot reactivity with BP240 (see Fig. $5 \mathrm{~A}$ ).

Immunolocalization of the BP180 antigen was performed with the use of the rabbit anti-BP180 FP. These antibodies labeled the BMZ of human epidermal cryosections producing a linear staining by indirect IF and giving a polar staining of trypsin-dissociated epidermal basal cells (data not shown). These immunocytochemical staining patterns obtained with the rabbit anti-BP180 FP are indistinguishable from the staining patterns produced by human BP sera $(3,5,6)$. The rabbit antiserum was also analyzed by immuno-EM on ultrathin sections of human skin using the indirect immunoperoxidase method. This analysis demonstrated that the immunoreactants were localized to the region of the epidermal hemidesmosome, overlapping the basal cell membrane. As previously reported from our laboratory as well as others, a very similar immuno-EM staining pattern is also produced by human BP sera $(5,7,8,22)$. A more precise immunolocalization of the 

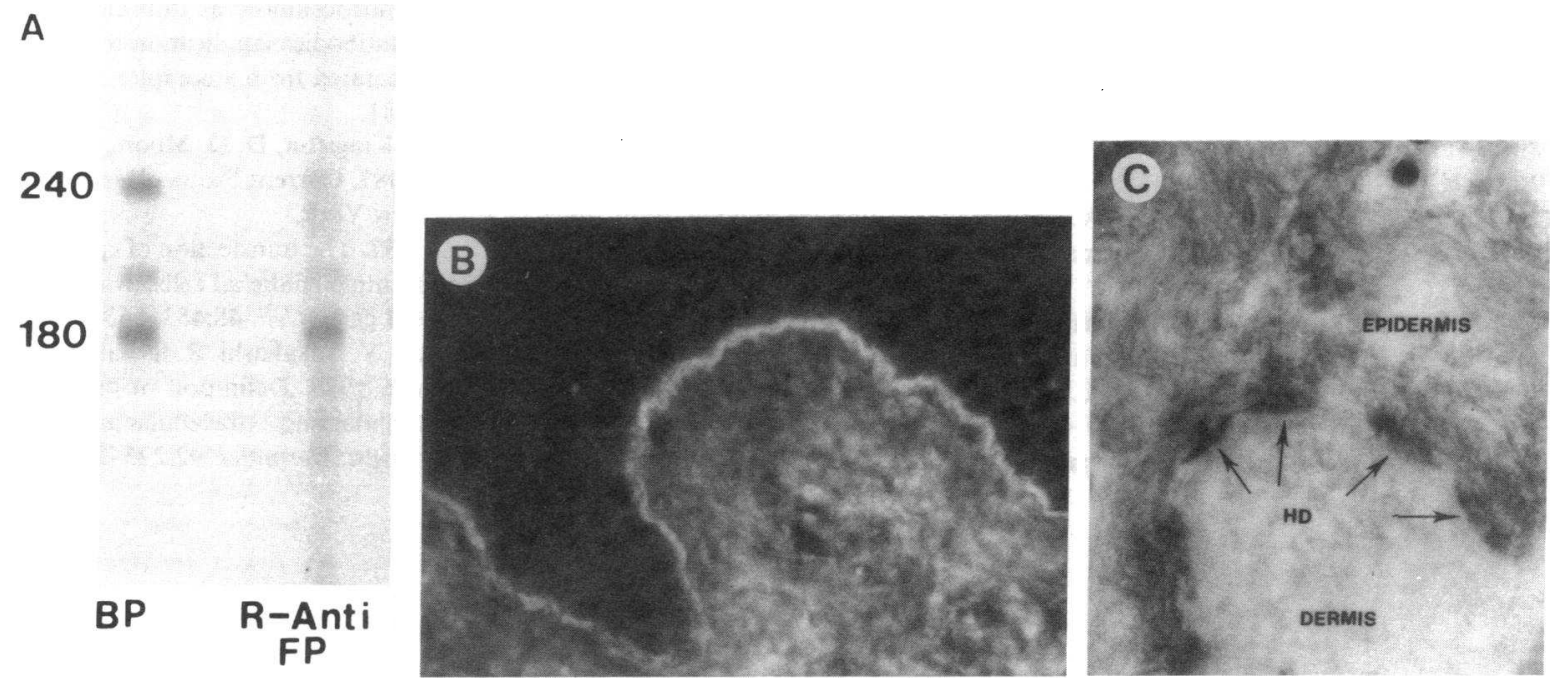

Figure 5. Rabbit antibodies prepared against BP180 fusion protein react with the BP180 antigen and label the epidermal hemidesmosome. $(A)$ Immunoblot analysis of a total protein extract from human epidermis probed with human BP serum (lane 1 ) and rabbit antibodies prepared against gel-purified lambda-BP180 FP (lane 2). (B) A cryosection of normal human skin was labeled by indirect IF using the rabbit antiserum directed against the BP180 FP. The observed linear staining of the BMZ is indistinguishable from the staining pattern obtained with BP sera. (C) Ultrathin section of normal human skin labeled with the rabbit anti-BP180 FP antiserum using an indirect immunoperoxidase staining procedure. Deposits of the electron-dense enzymatic reaction product are specifically associated with the hemidesmosomes on the basal surface of the basal keratinocytes.

rabbit anti BP180 FP antibodies to hemidesmosomal structures are presently underway.

The major conclusions which can be drawn from this molecular genetic analysis of the major BP antigens are as follows. The BP180 and BP240 antigens are the products of distinct RNA transcripts. The cloned portion of the BP180 and BP240 antigens do not contain common epitopes, i.e., each of the two cDNA-encoded FPs is antigenically distinct by immunoblotting. $44 \%$ of BP sera and $88 \%$ of $\mathrm{HG}$ sera recognized epitopes which are located within the stretch of $\sim 200$ amino acids encoded by the lambda-BP180 cDNA. And finally, the BP180 antigen has been localized to the epidermal hemidesmosome.

\section{Acknowledgments}

The authors acknowledge the invaluable advice and support of Dr. W. C. Earnshaw of the Department of Cell Biology, The Johns Hopkins University. Superb technical support was provided by Ms. H. Santana and Ms. P. Elias.

Supported in part by U. S. Public Health Service Grants R01AR32599, R01-AR32081, R01-AR32490, R01-GM35212, and K04AR01686 from the National Institutes of Health and a grant from the Dermatology Foundation.

\section{References}

1. Lever, W. F. 1965. Pemphigus and Pemphigoid. Charles C. Thomas Publisher, Springfield, IL. 75-103.

2. Gianotti, B., P. Fabbri, and E. Panconesi. 1975. Ultrastructural findings in bullous pemphigoid. J. Cutaneous Pathol. 2:103-108.

3. Jordon, R. E., E. H. Beutner, E. Witebsky, G. Blumenthal, W. L. Hale, and W. F. Lever. 1967. Basement membrane zone antibodies in bullous pemphigoid. J. Am. Med. Assoc. 200:751-756.

4. Holubar, K., K. Wolff, K. Konrad, and E. H. Beutner. 1975.
Ultrastructural localization of immunoglobulins in bullous pemphigoid skin. J. Invest. Dermatol. 64:220-227.

5. Mutasim, D. F., Y. Takahashi, R. S. Labib, G. J. Anhalt, H. P. Patel, and L. A. Diaz. 1985. A pool of bullous pemphigoid antigen(s) is intracellular and associated with the basal cell cytoskeleton-hemidesmosome complex. J. Invest. Dermatol. 84:47-53.

6. Takahashi, Y., D. F. Mutasim, H. P. Patel, G. J. Anhalt, R. S. Labib, and L. A. Diaz. 1985. The use of human pemphigoid autoantibodies to study the fate of epidermal basal cell hemidesmosomes after trypsin dissociation. J. Invest. Dermatol. 85:309-313.

7. Westgate, G. E., A. C. Weaver, and J. R. Couchman. 1985. Bullous Pemphigoid antigen localization suggests an intracellular association with hemidesmosomes. J. Invest. Dermatol. 84:218-224.

8. Jones, J. C. R., K. M. Yokoo, and R. D. Goldman. 1986. Is the hemidesmosome a half desmosome? An immunological comparison of mammalian desmosomes and hemidesmosomes. Cell Motil. Cytoskeleton. 6:560-569.

9. Stanley, J. R., P. Hawley-Nelson, S. H. Yuspa, E. M. Shevach, and S. I. Katz. 1981. Characterization of bullous pemphigoid antigen: a unique basement membrane protein of stratified squamous epithelia. Cell. 24:897-903.

10. Mueller, S. M., V. Klaus-Kovtun, and J. R. Stanley. 1989. A 230-kD basic protein is the major bullous pemphigoid antigen. $J$. Invest. Dermatol. 92:33-38.

11. Labib, R. S., G. J. Anhalt, H. P. Patel, D. F. Mutasim, and L. A. Diaz. 1986. Molecular heterogeneity of the bullous pemphigoid antigens as detected by immunoblotting. J. Immunol. 136:1231-1235.

12. Bernard, P., L. Didierjean, F. Denis, J-H. Saurat, and J-M. Bonnetblanc. 1989. Heterogeneous bullous pemphigoid antibodies: detection and characterization by immunoblotting when absent by indirect immunofluorescence. J. Invest. Dermatol. 92:171-174.

13. Morrison, L. H., R. S. Labib, J. J. Zone, L. A. Diaz, and G. J. Anhalt. 1988. Herpes gestationis autoantibodies recognize a $180 \mathrm{kD}$ human epidermal antigen. J. Clin. Invest. 81:2023-2026.

14. Lawley, T. J., G. Stingle, and S. I. Katz. 1978. Fetal and maternal factors in herpes gestationis. Arch. Dermatol. Res. 114:552-556.

15. Stanley, J. R., T. Tanaka, S. Mueller, V. Klaus-Kovtun, and D. Roop. 1988. Isolation of a complementary DNA for bullous pemphi- 
goid antigen by use of patients' autoantibodies. J. Clin. Invest. 82:1864-1870.

16. Young, R. A., and R. W. Davis. 1983. Efficient isolation of genes using antibody probes. Proc. Natl. Acad. Sci. USA. 80:11941198.

17. Earnshaw, W. C., K. F. Sullivan, P. H. Machlin, C. A. Cooke, D. A. Kaiser, T. D. Pollard, N. F. Rothfield, and D. W. Cleveland. 1987. Molecular cloning of CDNA for CENP-B, the major human centromere autoantigen. J. Cell Biol. 104:817-829.

18. Miller, H. 1987. Practical aspects of preparing phage and plasmid DNA: growth, maintenance, and storage of bacteria and bacteriophage. In Guide to Molecular Cloning Techniques. S. L. Berger and A. R. Kimmel, editors. Methods Enzymol. 152:145-170.

19. Sheng, H. Z., J. Hoogenraad, P. R. Carnegie, and C. C. A.
Bernard. 1987. Use of protein-bearing nitrocellulose as immunogen for in vitro production of monoclonal antibodies: application to myelin basic protein electrophoretically separated from a complex brain protein mixture. Immunol. Lett. 16:75-81.

20. Ausubel, F. M., R. Brent, R. E. Kingston, D. D. Moore, J. G. Seidman, J. A. Smith, and K. Struhl. 1987. Current Protocols in Molecular Biology. John Wiley \& Sons, New York.

21. Giudice, G. J., and E. Fuchs. 1987. The transfection of epidermal keratin genes into fibroblasts and simple epithelial cells: evidence for inducing a type I keratin by a type II gene. Cell. 48:453-563.

22. Mutasim, D. F., L. H. Morrison, Y. Takahashi, R. S. Labib, J. Skouge, L. A. Diaz, and G. A. Anhalt. 1989. Definition of bullous pemphigoid antibody binding to intracellular and extracellular antigen associated with hemidesmosomes. J. Invest. Dermatol. 92:225-230. 